\title{
Frequency-Domain GLR Detection of a Second-Order Cyclostationary Signal Over Fading Channels
}

\author{
Jaume Riba, Senior Member, IEEE, Josep Font-Segura, Student Member, IEEE, Javier Villares, Member, IEEE, \\ and Gregori Vázquez, Senior Member, IEEE
}

\begin{abstract}
Cyclostationary processes exhibit a form of frequency diversity. Based on that, we show that a digital waveform with symbol period $T$ can be asymptotically represented as a rank-1 frequency-domain vector process which exhibits uncorrelation at different frequencies inside the Nyquist spectral support of $1 / T$. By resorting to the fast Fourier transform (FFT), this formulation obviates the need of estimating a cumbersome covariance matrix to characterize the likelihood function. We then derive the generalized likelihood ratio test (GLRT) for the detection of a cyclostationary signal in unknown white noise without the need of a assuming a synchronized receiver. This provides a sound theoretical basis for the exploitation of the cyclostationary feature and highlights an explicit link with classical square timing recovery schemes, which appear implicitly in the core of the GLRT. Moreover, to avoid the well-known sensitivity of cyclostationary-based detection schemes to frequency-selective fading channels, a parametric channel model based on a lower bound on the coherence bandwidth is adopted and incorporated into the GLRT. By exploiting the rank1 structure of small spectral covariance matrices, the obtained detector outperforms the classical spectral correlation magnitude detector.
\end{abstract}

Index Terms-GLRT, LMPIT, Cognitive Radio, Cyclostationarity Based Detection, Spectral Correlation, Timing Synchronization, Frequency-Smoothed Cyclic Periodogram.

EDICS-SPC-DETC Detection, estimation and demodulation.

\section{INTRODUCTION}

To efficiently use the spectrum and manage the interference caused to Primary Users (PU), interweave cognitive receivers [1] must reliably detect the transmission by the PU under challenging situations, such as low signal-to-noise ratio (SNR), shadowing and fading. Motivated by this requirement, the problem of detecting the presence of a PU signal in white noise on a given band has gained considerable attention in the last few years. A variety of approaches have been developed to tackle this basic problem, and their structure depends on the degree of side information that cognitive users have about the primary-to-secondary propagation wireless channel.

J. Riba, J. Font-Segura, J. Villares, and G. Vazquez are within the Signal Theory and Communications Department of the Technical University of Catalonia (UPC), Jordi Girona 1-3, Campus Nord D5-\{116, 214, 115, 204\}, 08034 Barcelona, Spain, \{jaume.riba, josep.font-segura, javier.villares, gregori.vazquez\}@upc.edu.

This work has been partially funded by the Spanish Government under TEC2010-21245-C02-01 (DYNACS), CONSOLIDER INGENIO CSD200800010 (COMONSENS), CENIT CEN-20101019 (THOFU), and the Catalan Government (AGAUR) under Grant 2009SGR1236 and Fellowship FI-2010.
Higher degrees of side information on the noise and PU signal models induce to detectors with enhanced capabilities, yet at the additional cost of higher sensitivity to model inaccuracies.

Concerning the noise component, for instance, uncertainties on the white noise floor give rise to an SNR wall below which a detector will fail to be robust [2]. In a more general situation, the aggregated noisy component may be composed of narrow or wide band co-channel interferences (i.e., signals other than the PU signal that cognitive users are not interested on detecting). For example, interfering orthogonal frequency division multiplexing (OFDM) signals propagating through multi-path channels can be modeled as colored wide-sense stationary (WSS) Gaussian noise [3]. In this line, cyclostationarity feature detection has emerged, among other reasons, as a means for relaxing assumptions on noise statistics [4], thus gaining robustness against changing noise levels and interference activity. As an example, when the noise variance is unknown, it is shown that feature outperforms energy detector [5].

Concerning the received PU component, the adoption of a model is also relevant for designing the detection strategy, and it includes both transmitted PU signal model and propagation channel model. The accuracy in the modeling of the transmitted PU signal can range from simply knowing the potential occupied band to exactly knowing the transmitted signal waveform (e.g., PU pilot signals). In the middle of these two extreme cases one can make use of other features such as the transmitted power spectral density [6] (including or not the signal power), cyclostationarity, modulation type, etc. [1].

Regarding the propagation channel, the degree of frequencyselectivity associated to a particular wireless scenario can be modeled by the effective length of the channel response or, equivalently, by the channel coherence bandwidth [7]. Likewise, the channel coherence time can be used to model the time-varying nature. In practice, the time-varying behavior of a channel may arise due either to time fluctuations on the received PU signal power and/or to the presence of clock drifts, jitters and variable timing offsets due to errors on the receiver sampling clock and/or carrier frequency. Depending on how the detection algorithms are equipped with signal features, their performance and sensitivity to the propagation channel conditions will vary. For example, if the knowledge of the transmitter PU spectrum is exploited, the carrier frequency error can produce a frequency shift on it; if cyclostationary features are used, the frequency selective nature of the channel 
may partially or completely kill them [8]; if synchronized averaging is required (for example to exploit the knowledge of cyclic frequencies), the time clock drift can put a limitation on the averaging time [9], etc.

The propagation channel depends also on whether multiple antennas are used at the receiver, and they have been proposed by various researchers as a means to improve the PU detection performance [10], [11], [12]. Assuming Gaussian signals and noise (a commonly used hypothesis leading to tractable analysis and useful detectors) and depending on the degree of structure associated to the noise models, different tests arise such as a test for block diagonal structure of the covariance matrix in the most general case or an sphericity test for i.i.d. noises (see for instance [12] and references therein). On the other hand, the knowledge on the rank of the covariance matrix has been shown to play a relevant role in the modeling of the PU signal. While a rank-1 spatial covariance matrix structure for the signal of interest is considered in [13], a generalization for covariance matrices with any known rank is derived in [11], [14]. The exploitation of the low rank nature of the PU signal helps, for instance, in the noise floor estimation process, as one has access to a noise subspace which is free of signal component. As, in the general case, the likelihood depends on unknown parameters, the generalized likelihood ratio test (GLRT) has been considered by most approaches, as well as the locally most powerful invariant test (LMPIT) when the particular but challenging case of close hypothesis is focused, [15].

In the single antenna case, the exploitation of the signal and noise structure requires the specification of models for the temporal autocorrelation function of these signals. In the case of WSS signals, asymptotic (large-data-record) frequencydomain approximations of the likelihood ratio have been derived in [16] by resorting to asymptotic properties of Toeplitz matrices, showing that detectors can be formulated from the signal periodogram, smoothed by an appropriately selected spectral mask. This approach yields to significant saving on computational complexity, which is specially interesting in order to make cognitive radios feasible. In the case of a second order cyclostationary signal, the particular structure of the covariance matrix can be exploited in the time-domain as well as in the frequency-domain (spectral correlation property) to either improve the detection performance or as the unique feature to distinguish the signal from noise.

The main goal of this paper is to formulate the singleantenna detection problem of a second order stationary signal by resorting to and taking benefit from the mature and recent detection theory advances developed in the field of array processing, providing an original formulation of cyclostationarybased detection. Although the proposed approach can also be used with multiple antennas (MIMO techniques), the single antenna case is chosen as the main framework in order to gain clarity and concision in the exposition. By considering large data records as in [16], a frequency-domain approach is naturally obtained. We propose a vectorial frequency-domain processing that leads to a signal model which resembles the multi antenna model widely used for WSS signals, where the low rank information is specifically due to the cyclostationary nature of the PU signal. Particularly, the rank-1 structure for pulse-shaped digital modulations considered in our formulation plays the equivalent role of the spatial signature typically found in the array processing field.

This basic frequency-domain formulation was already introduced by the authors in [17] where the low rank nature of the spectral covariance matrix was exploited to derive unbiased SNR estimators based solely on second order statistics. As the GLRT tends asymptotically to be optimal in the NeymanPearson sense [15], it will be the adopted tool in this paper in order to extend the applicability of the vectorial frequencydomain approach to detection problems.

\section{A. Contributions}

In summary, the main contributions of this work are the following.

1) Vectorial frequency-domain processing: The proposed frequency-domain vectorial processing, motivated by the inherent asymptotic spectral correlation properties of cyclostationary processes, possibilities the use of well-known detection theory framework (developed so far in the field of array processing) to the singleantenna cyclostationary-based spectrum sensing field. The asymptotic log-likelihood function is given in (17). In fact, the proposed kind of data processing constitutes the root idea that enables the rest of contributions.

2) Exploitation of the pulse-shaped structure of digital modulations for detection. New detection schemes are obtained which are specific for the pulse-shaped digital modulations that are commonly used in most communication standards. As a direct consequence of the vectorial frequency-domain formulation, the pulse-shaped structure is simply manifested as a rank-1 property of a set of spectral covariance matrices. Under the authors' knowledge, the particular rank-1 structure of the spectral-correlation exhibited by pulse-shaped digital modulations has never been exploited for detection purposes. In that sense, we try to fill an apparent research gap in this direction, as it was done in [13] to exploit the rank-1 structure in the spatial counterpart.

3) Frequency-Domain Estimator-correlator: The asymptotic form of the estimator-correlator detector for pulseshaped digital modulations is obtained in (25)-(27), which generalizes that derived in [16] for stationary signals. Although the derived detector requires perfect channel state information (including the need for perfect timing synchronization), in the case of pulse-shaped digital modulations with modulation pulse free of intersymbol interference (ISI) under white noise, the knowledge of the SNR is not required by the new detector, in contrast with its classical stationary counterpart for which the hypothesis of low-SNR is typically adopted to cope with this issue, [6].

4) Scale-invariant GLRT detectors based on sub-band sample spectral covariances: Different forms of the GLRT detector for pulse-shaped digital modulations are derived (see (39), (45) and (53)), depending on the degree of side 
information about the propagation channel. It is shown that, in all cases, the sufficient statistic for detection consists on an estimate of a frequency-smoothed (consistent) version of the cyclic periodogram. New detectors based on the computation of the largest eigenvalue of small spectral covariance matrices are proposed, which are less computationally demanding that those based on the singular value decomposition of a huge correlation matrix. They show improved performance compared with classical cyclic-spectrum based approaches.

\section{B. Notation}

In the sequel, $[\mathbf{a}]_{i}$ and $[\mathbf{A}]_{i, j}$ denote the entries of a vector and a matrix, respectively, $\operatorname{diag}(\mathbf{A})$ is a diagonal matrix whose diagonal elements are equal to the diagonal elements of the square matrix $\mathbf{A}, \lambda_{\max }(\mathbf{A})$ denotes the maximum eigenvalue of a positive-definite square matrix, $\odot$ is the Hadamard (element-wise) product, $\operatorname{det}(\mathbf{A})$ denotes the determinant of A, $\mathbf{1}$ is a column vector with entries equal to $1,\lceil x\rceil$ and $\lfloor x\rfloor$ are the integer ceiling and floor of a real $x \geqslant 0$, respectively, $f(\mathbf{x})$ is a column vector whose entries are $f\left([\mathbf{x}]_{i}\right)$, where $f($. is a scalar function of scalar argument, $|\mathbf{a}|$ is a vector whose elements are $[|\mathbf{a}|]_{i}=\left|[\mathbf{a}]_{i}\right|,\|\mathbf{a}\|$ denotes the 2 -norm of a vector, $[a, b]$ and $[a ; b]$ are used to specify row and column vectors, respectively, $\mathbf{I}_{L}$ is the $L \times L$ identity matrix, and q.m. denotes quadratic mean convergence.

\section{Paper Structure}

The remainder of this paper is organized as follows. Section II poses the problem and provides some brief background on cyclostationarity. The basic formulation of the problem is shown in Section III by deriving the asymptotic likelihood function that will be used through the paper. The GLRT formulation is presented in sections IV and V, for the flat fading and frequency-selective fading, respectively. Section VI evaluates computational complexity of the proposed algorithms. Finally, the performance of the proposed detectors is illustrated by means of numerical simulations in Section VII, and Section XIII summarizes the main conclusions.

\section{Problem Statement AND BACKGRound}

Consider the binary hypothesis testing problem of detecting a pulse-shaped digital modulations in stationary noise

$$
\begin{aligned}
& \mathcal{H}_{0}: \quad y(t)=n(t) \\
& \mathcal{H}_{1}: \quad y(t)=n(t)+x(t),
\end{aligned}
$$

with

$$
x(t)=\sqrt{\gamma T} h(t) * \sum_{n} a[n] p(t-n T),
$$

where $*$ denotes convolution, $n(t)$ is WSS circular noise of power spectral density $S_{n}(f)=N_{0} / 2$ inside the signal band and zero outside (a situation that models an ideal antialiasing filter that suppresses noise and possible out of band interference), $h(t)$ is the (complex) propagation channel, and $\gamma>0$ is the signal strength. We assume that the symbols $a[n]$ are of unite variance and the modulation pulse $p(t)$ is of unite energy. The signal model in (2) is valid for a wide class ${ }^{1}$ of digital modulations in either single or multi carrier (OFDM) configurations, which includes all Pulse Amplitude Modulations (PAM) and Quadrature Amplitude Modulations (QAM) used for example in the digital video broadcast standard DVB-T2 [18], as well as the Amplitude Phase Shift Keying (APSK) format defined in the digital television broadcast standard DVB-S2 [19]. The two-sided bandwidth of the (complex envelope) digital modulation $x(t)$ is $B=(1+\alpha) / T$ where $\alpha>0^{2}$ is the excess bandwidth (roll-off parameter) and $T$ is the symbol interval.

As the asymptotic frequency-domain formulation of the detection problem will naturally lead to the concepts of cyclic spectral density (CSD) and frequency-smoothed cyclic periodogram (FS-CP), both are then briefly introduced here in order to make subsequent notation clear. The reader is referred to [20], [21], [22], [23], [24] for more details on this subject. The CSD of a process $x(t)$ is specifically defined in this paper as the Cross Spectral Density of the pair $x(t) e^{-j \pi \alpha t}$ and $x(t) e^{j \pi \alpha t}$, i.e.

$$
S_{x}^{\alpha}(f)=\lim _{T_{0} \rightarrow \infty} E\left[X_{T_{0}}\left(f+\frac{\alpha}{2}\right) X_{T_{0}}^{*}\left(f-\frac{\alpha}{2}\right)\right],
$$

where $X_{T_{0}}(f)$ is the normalized finite Fourier transform of a $T_{0}$-length realization of the process ${ }^{3}$,

$$
X_{T_{0}}(f)=\frac{1}{\sqrt{T_{0}}} \int_{-\frac{T_{0}}{2}}^{\frac{T_{0}}{2}} x(t) e^{-j 2 \pi f t} d t .
$$

It is well-known [25], that for a fixed value of $f$, the random variable $X_{T_{0}}(f)$ has asymptotic normality for $T_{0} \rightarrow \infty$, when the signal samples $x\left(t_{1}\right)$ and $x\left(t_{2}\right)$ are independent for sufficiently large values of $\left|t_{1}-t_{2}\right|$. The implication of this fact is that if $E\left[X_{T_{0}}\left(f_{1}\right) X_{T_{0}}^{*}\left(f_{2}\right)\right] \rightarrow 0$, then the pair of random variables $X_{T_{0}}\left(f_{1}\right)$ and $X_{T_{0}}\left(f_{2}\right)$ has asymptotic independence. This fact will be fully exploited through this paper to simplify the expression of the likelihood functions and then reduce the computational complexity of the derived cyclic spectrum based detectors.

The cyclic periodogram (CP) of $x(t)$ is defined as the cross periodogram of the pair $x(t) e^{-j \pi \alpha t}$ and $x(t) e^{j \pi \alpha t}$, i.e.

$$
I_{x, T_{0}}^{\alpha}(f)=X_{T_{0}}\left(f+\frac{\alpha}{2}\right) X_{T_{0}}^{*}\left(f-\frac{\alpha}{2}\right),
$$

and it can be seen as an inconsistent estimate of $S_{x}^{\alpha}(f)$. The FS-CP is defined as

$$
\hat{S}_{x, T_{0}, \Delta f}^{\alpha}(f)=\frac{1}{\Delta f} \int_{f-\frac{\Delta f}{2}}^{f+\frac{\Delta f}{2}} I_{x, T_{0}}^{\alpha}\left(f^{\prime}\right) d f^{\prime},
$$

\footnotetext{
${ }^{1}$ The exception for which the proposed approach cannot be applied is for the class of Continuous Phase Modulations (CPM), were the information symbols and the transmitted waveform are not linearly related.

${ }^{2}$ Although for classical root raised cosine (RRC) pulses it holds that $0<$ $\alpha \leq 1$, i.e., the excess bandwidth is not higher than $100 \%$ of the Nyquist rate $1 / T$, our study is general for any value of $\alpha$ in order to accommodate wideband modulation schemes as well.

${ }^{3}$ For the sake of clarity, continuous-time Fourier transforms are used in this paper. However, in practice, the derived detection schemes will employ the Fast Fourier Transform (FFT) based on the received signal samples taken at a minimum sampling rate of $f_{s, \text { min }}=(1+2\lceil\alpha\rceil) / T$. Other simpler sufficient conditions for the sampling rate (see for instance [24], Eq. (3)) would require that $f_{s, \text { min }}=2(1+\lceil\alpha\rceil) / T$. In particular, the number of samples per symbol would be $N_{s s}=4$ for $0<\alpha \leq 1$ and $N_{s s}=8$ for $\alpha=3$, which will be the cases considered in the simulation results.
} 
which constitutes a consistent estimate of the CSD in the sense that

$$
S_{x}^{\alpha}(f) \stackrel{q . m .}{=} \lim _{\Delta f \rightarrow 0} \lim _{T_{0} \rightarrow \infty} \hat{S}_{x, T_{0}, \Delta f}^{\alpha}(f),
$$

where the order of the two limits cannot be interchanged. Finally, for a process defined as in (2), and assuming, without loss of generality, stationary zero-mean (real or complex) uncorrelated data symbols, the CSD is given by

$$
S_{x}^{l / T}(f)=\gamma G\left(f+\frac{l}{2 T}\right) G^{*}\left(f-\frac{l}{2 T}\right),
$$

where $l$ is an integer and

$$
G(f)=H(f) P(f),
$$

with $H(f)$ and $P(f)$ the channel frequency response and modulation pulse Fourier transform, respectively. We note that the factorization of the CSD as indicated in Eqs. (8) and (9), which holds even in the presence of a frequency-selective channel $H(f)$, is a consequence solely of the pulse-shaped structure of the digital modulations modeled in Eq. (2), and it constitutes the core idea behind the exploitation of the rank-1 structure developed later on ${ }^{4}$.

If the process $x(t)$ is WSS, then for large $T_{0}$ the process $X(f)$ becomes asymptotically Gaussian ${ }^{5}$ (by invoking the central limit theorem, CLT) and processes $X(f+\delta)$ and $X(f)$ are asymptotically uncorrelated (i.e., independent) for $\delta \neq 0$. This property is well-known (see for instance [21]) and forms the basis for a frequency-domain signal processing involving detection and estimation [16]. If the process is not WSS, then $Y(f)$ (i.e. the Fourier transform of $y(t)$ in Eq. (1)) has some degree of spectral correlation, [4]. In the case of pure cyclostationary processes, the spectral correlation may be non-zero only for values of $\delta$ which are multiples of the fundamental cyclic period $(1 / T)$ and, at the same time, lower than the signal bandwidth ( $B$, i.e., the overall spectral support) of the complex base-band signal.

\section{ASYMPTOTIC FREQUENCY-Domain LIKELIHOOD FunCTION OF A CYCLOSTATIONARY PROCESS}

Consider the normalized Fourier transform of a $M T$-length ${ }^{6}$ realization of a process $y(t)$, i.e., $Y_{M T}(f)$. The approach proposed in this paper consists basically on introducing a vectorial treatment of the frequency-domain process $Y_{M T}(f)$ such that we can still rely on some asymptotically uncorrelated

\footnotetext{
${ }^{4} \mathrm{We}$ remark that Eq. (8), which is the core of this paper, is valid as well in the presence of correlated data symbols (e.g. coded modulations). The rationale is that if the stationary stream $a[n]$ has correlation $r_{a}[m]=E\left[a[n+m] a^{*}[n]\right]$ (which admits some factorization in the form $\left.r_{a}[m]=b[m] * b^{*}[-m]\right)$, it can be modeled as $a[n]=a^{\prime}[n] * b[n]$, where $a^{\prime}[n]$ is an equivalent uncorrelated stream. Then, Eq. (2) becomes $x(t)=\sqrt{\gamma T} h^{\prime}(t) * \sum_{n} a^{\prime}[n] p(t-n T)$ where $h^{\prime}(t)$ is an equivalent channel given by $h^{\prime}(t)=\sum_{m} b[m] h(t-m T)$, thus proving the general validity of Eq. (8), with $G(f) \stackrel{m}{=} H^{\prime}(f) P(f)$ and $H^{\prime}(f)$ the frequency response of $h^{\prime}(t)$.

${ }^{5}$ The reader is referred to [26] for a further rationale on the Gaussian assumption in the context of digital modulation signals.

${ }^{6}$ Samples of partially received blocks at the beginning and the end are discarded. These end-effects will be very small for $M \gg 1$. A similar consideration is taken, for instance, by the time-domain approach in [9].
}

domain to simplify the detection problem. To make that clear, we define a frequency-domain observation vector as

$$
\mathbf{y}(v) \triangleq Y_{M T}\left(\mathbf{f}_{v}\right),
$$

where $\mathbf{f}_{v}$ is a $L$-dimensional frequency-domain uniform sampling vector defined as

$$
\mathbf{f}_{v}=v \mathbf{1}+\mathbf{s}
$$

where

$$
\mathbf{s}=\frac{1}{T}\left(\begin{array}{c}
(L-1) / 2 \\
(L-1) / 2-1 \\
\vdots \\
-(L-1) / 2
\end{array}\right)
$$

i.e., $\left[\mathbf{f}_{v}\right]_{l}=v_{l}=v+(L-1) /(2 T)-(l-1) / T$, where $L=\lceil B T\rceil$ is the dimension of the observation vector and $v$ is an auxiliary frequency within the range $-1 /(2 T) \leq$ $v \leq 1 /(2 T)^{7}$. We note that the signal bandwidth to symbol rate ratio determines the dimension of the observation vector ${ }^{8}$, while the range of the auxiliary frequency $v$ is held constant. For example, in the important case of digital modulations with no more than $100 \%$ of excess bandwidth, we have simply that $L=2$ and the frequency-domain observation vector becomes

$$
\mathbf{y}(v)=\left(\begin{array}{c}
Y_{M T}\left(v_{+}\right) \\
Y_{M T}\left(v_{-}\right)
\end{array}\right)
$$

where $v_{+}=v+1 /(2 T), v_{-}=v-1 /(2 T)$. This specific case was considered by the authors in [17] to derive a SNR estimator based on second order statistics, and our aim here is to generalize the applicability of the approach to a wider class of problems.

As a direct consequence of the asymptotic spectral correlation property indicated in Eq. (3), and due to the wide sense stationarity of the additive noise term, the vector process $\mathbf{y}(v)$ adopts the following second order statistics:

$$
E\left\{\mathbf{y}(v+\delta) \mathbf{y}^{H}(v)\right\} \rightarrow\left\{\begin{array}{cc}
\mathbf{S}_{\mathbf{y}}(v) & \delta=0 \\
\mathbf{0} & \delta \neq 0
\end{array},\right.
$$

where the $L \times L$ spectral matrix $\mathbf{S}_{\mathrm{y}}(v)$ is defined as

$$
\left[\mathbf{S}_{\mathrm{y}}(v)\right]_{i, j \in\{1, \ldots, \mathrm{L}\}}=S_{y}^{v_{i}-v_{j}}\left(\frac{v_{i}+v_{j}}{2}\right),
$$

and $S_{y}^{\beta}(v)$ is the CSD of $y(t)$. As a result, by introducing a vectorial (instead of scalar) spectral process to treat pure cyclostationary processes, we succeed in recovering the uncorrelation property (although on an auxiliary frequency-domain $v$ ) similar to the one manifested by WSS processes in the standard frequency-domain $f$. Thus, (14) constitutes the basis for the extension of the frequency-domain treatment of WSS processes to cyclostationary processes, just by extending the dimension of the frequency-domain process.

\footnotetext{
${ }^{7}$ Note that the minimum and maximum frequency values inside vector $\mathbf{f}_{v}$ are $\min _{l, v}\left[\mathbf{f}_{v}\right]=-L /(2 T)$ and $\max _{l, v}\left[\mathbf{f}_{v}\right]=L /(2 T)$. Its difference is equal to $L / T$, which corresponds to the overall signal spectral support, where $L$ is clearly the ceil of the relative excess band with respect to Nyquist band $(1 / T)$.

${ }^{8}$ The integer $L$ coincides in practice with the minimum number of samples per symbol required for a digital implementation.
} 
For example, for $L=2$, the spectral matrix reduces simply to

$$
\mathbf{S}_{\mathrm{y}}(v)=\left(\begin{array}{cc}
S_{y}\left(v_{+}\right) & S_{y}^{1 / T}(v) \\
S_{y}^{-1 / T}(v) & S_{y}\left(v_{-}\right)
\end{array}\right)
$$

In the general case, as $\mathbf{y}(v+\delta)$ and $\mathbf{y}(v)$ become asymptotically independent in the auxiliary frequency domain $v$ as shown in (14), its log-likelihood function $\left(\ln \left(p_{\mathbf{y} \mid \mathcal{H}_{i}}(\mathbf{y})\right)_{i=0,1}\right)$ adopts (up to irrelevant additive constants) the following asymptotic frequency-domain form

$$
L(\mathbf{y})=-\int_{1 / T}\left(\ln \operatorname{det}\left(\mathbf{S}_{\mathbf{y}}(v)\right)+\mathbf{y}^{H}(v) \mathbf{S}_{\mathrm{y}}^{-1}(v) \mathbf{y}(v)\right) d v
$$

The main advantage of the obtained expression is the fixed (independent from data size) and low dimensionality $(L \times L)$ of the involved spectral matrices, which provides mathematical simplicity compared with the time-domain approach typically involving the estimation (and even the singular value decomposition (SVD) in some cases) of cumbersome covariance matrices, like in [27] and [28]. Using (8), the spectral matrices under both hypothesis are given by

$$
\begin{array}{ll}
\mathcal{H}_{0}: & \mathbf{S}_{\mathbf{y}}(v)=\mathbf{D}(v)=\operatorname{diag}\left(S_{n}\left(\mathbf{f}_{v}\right)\right) \\
\mathcal{H}_{1}: & \mathbf{S}_{\mathrm{y}}(v)=\mathbf{D}(v)+\gamma \mathbf{g}_{\varepsilon}(v) \mathbf{g}_{\varepsilon}^{H}(v),
\end{array}
$$

where

$$
\begin{aligned}
\mathbf{g}_{\varepsilon}(v) & =\mathbf{g}(v) \odot \mathbf{w}_{\varepsilon} \\
\mathbf{g}(v) & =G\left(\mathbf{f}_{v}\right), \\
\mathbf{w}_{\varepsilon} & =e^{j 2 \pi \varepsilon \mathbf{s}},
\end{aligned}
$$

and $0 \leq \varepsilon<1$ is the time-delay (or timing) parameter, the dimension of the spectral matrices is $L \times L$, and $G(f)$ is given in (9).

It is emphasized that, as a result of the frequency-domain formulation of the likelihood function, the general problem posed in (1) has been rewritten as the specific problem posed in (18). For example, for $L=2$, we have $\mathbf{g}(v)=$ $\left[G\left(v_{+}\right) ; G\left(v_{-}\right)\right]$and $\mathbf{w}_{\varepsilon}=\left[e^{j \pi \varepsilon} ; e^{-j \pi \varepsilon}\right]$. The important feature of the model defined in (18) is that the signal component has a rank-1 structure as a result of (8), while the noise component is full-rank. This low-rank nature of the signal to be detected is what provides the original formulation of the detection of cyclostationary signals developed in the following sections.

Remark 1. It is assumed in this paper that the receiver has a perfect knowledge of the fundamental cyclic frequency of the PU signal. In practice, if the receiver has no means for establishing synchronization with some pilot channel of the PU user, the presence of a clock offset will cause a smearing of the spectral correlation properties [5], and this will impose an upper limit on the value of signal block length to be used for the detection task. A similar situation occurs in the presence of a time-varying channel, in which the averaging time required for estimating the relevant signal features is limited by the coherence time of the channel response. If this upper bound on the block length is not enough to achieve the target detection performance, then several independent signal blocks need to be used and the independent detection metrics should be finally averaged. For clarity reasons, this issue will not be taken into account in the presentation of the different approaches in this paper, i.e., the channel will be assumed essentially constant during a length of $M T$ seconds.

\section{GLR Detection of Pulse-Shaped Modulations OVER FLAT FADING CHANNELS}

Consider a flat fading channel such that

$$
\mathbf{g}_{\varepsilon}(v)=\sqrt{\gamma} \mathbf{p}_{\varepsilon}(v),
$$

where (assuming $H(f)=1) \gamma$ is some arbitrary gain and $\left\|\mathbf{p}_{\varepsilon}(v)\right\|^{2}=1$. In the sequel, we will derive the GLRT for this specific scenario. First, we will consider that the timing and the noise floor $\left(\varepsilon\right.$ and $N_{0}$ ) are known by the receiver, and will derive the estimator-correlator for that problem, because it provides insights to the structure of the GLRT. Next, we will consider $\varepsilon$ and $N_{0}$ as nuisance parameters and will derive the GLRT. Finally, we will provide some links with classical timing recovery schemes.

\section{A. Estimator-Correlator}

It is well-known that, in the case of white noise $\left(S_{n}(f)=\right.$ $\left.N_{0}\right)^{9}$, the asymptotic frequency-domain estimator correlator assuming stationary signals is given by the following frequency average of the received signal periodogram [16]

$$
\int_{B} K_{\text {stat }}(f)\left|Y_{M T}(f)\right|^{2} d v \stackrel{\mathcal{H}_{1}}{\underset{\mathcal{H}_{0}}{\gtrless}} \eta,
$$

where $\eta$ is the detection threshold which is set to satisfy a false alarm level ${ }^{10}$, and $K_{\text {stat }}(f)$ is the stationary frequencydomain kernel defined as

$$
K_{\text {stat }}(f)=\frac{\gamma|G(f)|^{2}}{N_{0}\left(N_{0}+\gamma|G(f)|^{2}\right)} .
$$

Using the tools developed in the previous section and resorting to Woodbury formula to express $\mathbf{S}_{\mathrm{y}}^{-1}(v)$, we can similarly obtain the cyclostationary rank-1 frequency-domain version of the estimator-correlator as

$$
\int_{T^{-1}} K_{c y c}(v)\left|\mathbf{w}_{\varepsilon}^{H} \mathbf{z}(v)\right|^{2} d v \underset{\mathcal{H}_{0}}{\stackrel{\mathcal{H}_{1}}{\gtrless}} \eta,
$$

where $\mathbf{z}(v)$ is generally given by

$$
\mathbf{z}(v)=\frac{1}{\|\mathbf{g}(v)\|} \mathbf{g}_{\varepsilon}^{*}(v) \odot \mathbf{y}(v)
$$

\footnotetext{
${ }^{9}$ The consideration of white noise is taken in this paper to improve the clarity of the presentation. In the case of colored noise, one should simply prewhiten the data.

${ }^{10}$ The detector (23) and all the detectors proposed in this paper are derived under a GLRT formulation. Hence, the optimality in the NeymanPearson sense is satisfied, i.e., the detection probability is maximized for a given false alarm level. Setting the detection threshold requires the statistical characterization of the detector under $\mathcal{H}_{0}$. Even though this is in general a hard problem which has only been addressed in some specific situations, one can resort to the Wilks' theorem [15] as a general anlytical tool to set the threshold.
} 
and $K_{c y c}(v)$ is the cyclostationary frequency-domain kernel defined as

$$
K_{c y c}(v)=\frac{\gamma\|\mathbf{g}(v)\|^{2}}{N_{0}\left(N_{0}+\gamma\|\mathbf{g}(v)\|^{2}\right)} .
$$

In general, either for stationary or cyclostationary signals, apart of requiring the knowledge of the noise floor, the estimator correlator requires also the knowledge of the strength $\gamma$ of the useful signal to construct the frequency-domain kernels that adequately weight the periodogram and cyclicperiodogram, respectively, of a filtered version of the received signal. When this happens, as knowing the strength of the own signal to be detected may become unrealistic, the low-SNR assumption is typically adopted to obtain a locally optimum detector [29].

However, the estimator-correlator applied to our rank-1 cyclostationary signal detection problem exhibits a particular property that makes the low-SNR assumption unnecessary to construct the detector, namely, if the channel is flat $(H(f)=$ $h$ ) and the modulation pulse of the pulse-shaped signal fulfills the Nyquist criterion, then

$$
\|\mathbf{g}(v)\|^{2}=\|\mathbf{g}\|^{2}, \forall v,
$$

which implies that the kernel $K_{c y c}(v)$ becomes constant with respect to $v$, and then the estimator-correlator can be implemented without knowing the signal strength. As a result, the likelihood ratio statistic for ISI-free pulse-shaped digital modulations simply reduces to ${ }^{11}$

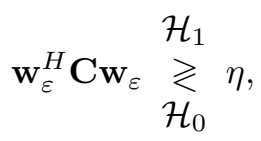

where $\mathbf{C}$ is the matched filter output sample covariance spectral matrix given by

$$
\mathbf{C}=\int_{T^{-1}} \mathbf{z}(v) \mathbf{z}^{H}(v) d v,
$$

and $\mathbf{z}(v)$ is the frequency-domain observation vector at the matched filter output, i.e.,

$$
\mathbf{z}(v)=\frac{1}{\|\mathbf{g}\|} \mathbf{g}^{*}(v) \odot \mathbf{y}(v)=\frac{1}{\|\mathbf{g}\|} Z_{M T}\left(\mathbf{f}_{v}\right) .
$$

The important remark is that the obtained detector is optimum at all values of SNR, without necessity of knowing the SNR a priori. Note that the entries of the above matrix $\mathbf{C}$ are equal to the area over intervals of length $1 / T$ along the frequencydomain of the estimated cyclic periodogram evaluated at the matched filter output. This basic idea will be further explored and generalized later on.

Although the previous key observation provides some advantage compared to the WSS case, we have still two important limitations that need to be solved: the need of assuming a synchronized receiver and the need of knowing the noise floor $N_{0}$ for the specification of the threshold. Even in the case that

\footnotetext{
${ }^{11}$ Despite that the rank-1 property leads to an implementation of the estimator-correlator without the knowledge of SNR, the specification of the threshold $\eta$ in (29) and the following, if one follows the Neyman-Pearson criterion, still requires this knowledge.
}

estimates of these parameters are available, sensitivity to their inaccuracies may be an issue in practice [2], [5]. Moreover, in a real situation one has to cope in addition with the issue of the sensitivity of the detector performance to the presence of an unknown frequency selective channel affecting the signal to be detected [8].

The subsequent sections are intended to extend the applicability of the proposed methodology to cope with the mentioned scenarios.

\section{B. GLR Detector}

The timing and the noise floor will now be considered as nuisance parameters whose Maximum Likelihood (ML) estimates under both hypothesis are required to construct the detector, and will focus on the case of ISI-free pulse-shaped digital modulations and white noise. For convenience, we define the signal support and the residual noise support as

$$
I_{s}=\left(-\frac{1}{2 T}, \frac{1}{2 T}\right)
$$

and

$$
I_{n}=\left(-\frac{\alpha-\lfloor\alpha\rfloor}{2 T}, \frac{\alpha-\lfloor\alpha\rfloor}{2 T}\right),
$$

respectively. The presentation of the main approach is best clarified by considering the SVD of the spectral coherence matrices

$$
\mathbf{S}_{\mathbf{y}}(v)=\mathbf{U}_{\varepsilon}(v) \boldsymbol{\Lambda}(v) \mathbf{U}_{\varepsilon}^{H}(v),
$$

with

$$
\mathbf{U}_{\varepsilon}(v)=\left[\mathbf{p}_{\varepsilon}(v), \mathbf{U}_{\varepsilon}^{\perp}(v)\right],
$$

and

$$
\boldsymbol{\Lambda}(v)=\left[\begin{array}{ccc}
N_{1} & \mathbf{0} & 0 \\
\mathbf{0} & N_{0} \mathbf{I}_{L-2} & \mathbf{0} \\
0 & \mathbf{0} & N_{0} \Upsilon(v)
\end{array}\right],
$$

where $\Upsilon(v)$ is an indicator function which takes 1 for $v \in I_{n}$ and 0 otherwise, $N_{1}=N_{0}+\gamma$ at $\mathcal{H}_{1}$ and $N_{1}=N_{0}$ at $\mathcal{H}_{0}$. $\mathbf{U}_{\varepsilon}^{\perp}(v)$ is a unitary matrix whose columns $\left(\mathbf{u}_{\varepsilon, l}(v)\right.$, $l=2, \ldots, L)$ span the orthogonal subspace of $\mathbf{p}_{\varepsilon}(v)$. The particularity of the eigenvalues of in (36) is that signal eigenvalue $N_{1}$ does not depend on $v$. The meaning is that the signal covariance eigenvalue multiplicity ${ }^{12}$ becomes maximum and equal to the Nyquist rate $(1 / T)$ for ISI-free digital modulations.

Note that $\left|I_{n}\right| \lesssim 1 / T$ only in the case that $\alpha \lesssim L-1$ and $\left|I_{n}\right| \gtrsim 0$ only in the case that $\alpha \gtrsim L-2$. This issue is considered in the definition of $\boldsymbol{\Lambda}(v)$ above by means of an indicator function $\Upsilon(v)$. The meaning is that, in general, the noise dimension is $L-1$ for $v \in I_{n}$ and $L-2$ otherwise. This is a consequence of assuming that the out-of-band noise has been removed by some filtering process. For example, in the case of the typical RRC pulses (see [17]) with $0<\alpha \leqslant 1$ the residual noise support would be $\left|I_{n}\right|=\alpha / T$.

\footnotetext{
${ }^{12} \mathrm{To}$ appreciate in more detail the relevance of this issue in the derivation of a GLRT, the reader is referred to [30] (and to its extension in [31]) where a unified framework for GLRT spectrum sensing of signals with known multiplicities is created, and to [9, Section III-A] for the exploitation of known eigenvalue multiplicities in the context of cyclostationary-based detection
} 
The formulation of the GLR detector involves the ML estimation of $N_{0}$ and $N_{1}$ under $\mathcal{H}_{0}$ and $\mathcal{H}_{1}$ from the loglikelihood function (17) based on the decomposition (34)-(36). After obtaining the estimates, substituting them into the loglikelihood function and removing data-independent constants (see Appendix A for the complete derivation), we obtain

$$
\max _{\varepsilon}\left[\frac{1}{\lambda_{\varepsilon}\left(1-\lambda_{\varepsilon}\right)^{\alpha}}\right] \underset{\mathcal{H}_{0}}{\stackrel{\mathcal{H}_{1}}{\gtrless}} \eta
$$

with

$$
\lambda_{\varepsilon}=\frac{\hat{P}_{s, \varepsilon}}{\hat{P}_{T}}
$$

which resembles that obtained in [32, Eq. (37)], in the array processing field. As $1 /(1+\alpha) \leq \lambda_{1} \leq 1$ and the function in the brackets in the above equation is monotonically increasing in this range, the final GLRT detector becomes

$$
\frac{\max _{\varepsilon}\left[\mathbf{w}_{\varepsilon}^{H} \mathbf{C w}_{\varepsilon}\right]}{\hat{P}_{T}} \underset{\mathcal{H}_{0}}{\stackrel{\mathcal{H}_{1}}{\gtrless}} \eta,
$$

which is simply the estimator-correlator detector maximized with respect to the timing parameter and then normalized by the total estimated power. Timing synchronization is then inherent to detection in the flat fading, white noise and ISI-free case, which has also been recognized in other time-domain GLRT approaches [31].

To get further insights into the detector (39) and, in addition, simplify the required search in $\varepsilon$, we observe that $\mathbf{w}_{\varepsilon}^{H} \mathbf{C w}_{\varepsilon}$ is a linear weighted combination of periodic terms in $\varepsilon$ within $0 \leq \varepsilon<1$ in the form $e^{j 2 \pi p \varepsilon}$, with $0 \leq p \leq L-1$. Therefore, by choosing (at least) $2 L$ uniform sampling values of the parameter $\varepsilon$, we can write the Fourier series

$$
\mathbf{w}_{\varepsilon}^{H} \mathbf{C w}_{\varepsilon}=2 \operatorname{Re}\left[\sum_{p=0}^{L-1} a_{p} e^{j 2 \pi p \varepsilon}\right],
$$

where $a_{p}$ is given by

$$
a_{p}=\frac{1}{2 L} \sum_{q=0}^{2 L-1} \mathbf{w}_{\frac{q}{2 L}}^{H} \mathbf{C w}_{\frac{q}{2 L}} e^{-j 2 \pi \frac{p q}{2 L}} .
$$

As a result, the maximization process can be avoided by exploiting the following approximation that allows to reduce the computational complexity:

$$
\max _{\varepsilon}\left[\mathbf{w}_{\varepsilon}^{H} \mathbf{C w}_{\varepsilon}\right] \approx \operatorname{tr}(\mathbf{C})+2 \sum_{p=1}^{L-1}\left|a_{p}\right|,
$$

where the right term becomes an equality in the particular case of $L \leq 2$, and an upper-bound in the general case. Based on the previous result, the final detector becomes

$$
\frac{1}{\hat{P}_{T}} \operatorname{tr}(\mathbf{C})+\frac{2}{\hat{P}_{T}} \sum_{p=1}^{L-1}\left|a_{p}\right| \underset{\mathcal{H}_{0}}{\stackrel{\mathcal{H}_{1}}{\gtrless}} \eta .
$$

whose validity will be analyzed through computer simulations. While the left hand side of the detector does not take into account the cyclostationarity property, the right hand side is a multi cycle feature detector that exploits cyclostationarity. The final detector is a combination of these two detection measures. This issue is further clarified in the next subsection.

\section{Time-Domain GLRT Implementation and Relationship with Square Timing Recovery Schemes}

Before going further, we explore some links with known results on timing synchronization to get insights into the core of the proposed detector in (39). In the case of $L=2$, the numerator of the GLRT can be expanded as

$$
\begin{aligned}
& \frac{1}{M T} \int_{I_{s}}\left[\left|Z_{M T}\left(v_{+}\right)\right|^{2}+\left|Z_{M T}\left(v_{-}\right)\right|^{2}+\right. \\
& \left.2 \operatorname{Re}\left\{e^{-j 2 \pi \varepsilon} Z_{M T}\left(v_{+}\right) Z_{M T}^{*}\left(v_{-}\right)\right\}\right] d v .
\end{aligned}
$$

Substituting $Z(f)$ as a function of $z(t)$ (matched filter output) in the previous result and maximizing with respect to $\varepsilon$ yields the following time-domain GLRT

$$
\frac{\int_{M T}|z(t)|^{2} d t+\left.2\left|\int_{M T}\right| z(t)\right|^{2} e^{-j 2 \pi \frac{t}{T}} d t \mid}{\int_{M T}|y(t)|^{2} d t} \underset{\mathcal{H}_{0}}{\underset{\mathcal{H}_{1}}{\gtrless}} \eta .
$$

The denominator of the previous detector is an estimate of the power at the matched filter input (total received power), and it provides overall invariance to scaling. The left hand side of the numerator is the estimated power at the matched filter output. The ratio between this term and the denominator is sensitive only to the fact that the spectrum of the signal is non flat (contrary to the noise) and, in that sense, it constitutes a measure of the amount of spectrum matching, ignoring completely the cyclostationary feature of the signal. Contrarily, the right hand side of the numerator is the modulus of the regenerated spectral line at the symbol rate at the modulus-squared matched filter output. The ratio between this term and the denominator is a feature detector, [27], [33], which is only sensitive to the presence of cyclostationarity by looking at the normalized strength of regenerated spectral line. The phase of the mentioned spectral line is just the timing estimate based on the classical squared timing recovery scheme, [34], [35], whose asymptotic equivalence with ML estimation was established in [36]. The final detector can be seen as a weighted combination of both statistics mentioned above.

Finally, it is emphasized that the common low-SNR assumption has not been required to derive a closed-form GLRT expression, contrarily to other classical cyclostationarity-based detection schemes, [4], [3]. This is solely due to the rank-1 structure of the frequency-domain vector and to the fact that $\|\mathbf{g}(v)\|$ is constant with $v$.

\section{GLR Detection of Pulse-Shaped Digital Modulations OVER FREQUENCY-SELECTIVE FADING CHANNELS}

Cyclostationary-based detection schemes are known to suffer from severe performance degradation in the presence of unknown frequency-selective channels [8], because the channel can attenuate or even kill the own specific signal cyclostationary feature which is exploited for its detection. 
However, when a frequency-selective channel affects a pulseshaped modulation, the resulting signal preserves the factorization property indicated in Eq. (8) for these kind of signals, with a modified modulation pulse according with Eq. (9). This means that the rank-1 structure is still present in the received waveform as indicated in Eq. (18), even in the presence of an unknown channel, because the presence of the channel only modifies the received pulse shape as seen in Eq. (9) without destroying the factorization property of the CSD in Eq. $(8)^{13}$. Based on that observation, the objective of this section is to develop the means for the exploitation of the rank-1 structure of the signal in a manner similar to that developed for the flat fading case, but capable of providing some degree of robustness to the channel response behavior by introducing more nuisance parameters into the GLRT formulation.

In the case of a frequency-selective channel $H(f)$ the received modulation pulse is modified according to the following expression

$$
\mathbf{g}(v)=\sqrt{\gamma(v)} \mathbf{h}(v)
$$

where without loss of generality we assume that $\|\mathbf{h}(v)\|=1$ and $\gamma(v)$ is some frequency-dependent arbitrary gain. The presence of $\mathbf{h}(v)$ (which models the combination of the modulation pulse and the channel) causes $\|\mathbf{g}(v)\|$ to be no longer constant in the auxiliary domain $v$ (as actually was in the flat fading case). Then, if a lower bound on the coherence bandwidth of the channel is known in the form of $B_{c}>1 /(K T)$ with $K$ some integer, we will have that $\left\|\mathbf{g}\left(v_{1}\right)\right\| \approx\left\|\mathbf{g}\left(v_{2}\right)\right\|$ for $\left|v_{1}-v_{2}\right| \ll B_{c}$ and $\left\|\mathbf{g}\left(v_{1}\right)\right\| \neq\left\|\mathbf{g}\left(v_{2}\right)\right\|$ for $\left|v_{1}-v_{2}\right| \gg B_{c}$. Based on the previous rationale, a $K$-parametric model based on the knowledge of $B_{c}$ is proposed based on

$$
\mathbf{g}(v)=\sqrt{\gamma_{k}} \mathbf{h}_{k}, \quad v \in I_{k}
$$

where we define

$$
N_{k}=N_{0}+\gamma_{k}, \quad v \in I_{k}
$$

and

$$
I_{k}=\left(\frac{-1 / 2+(k-1) / K}{T}, \frac{-1 / 2+k / K}{T}\right) .
$$

Note that the key assumption in the previous model is that every entry of the frequency vector $\mathbf{g}(v)$ remains essentially constant for values of $v$ which are within the coherence bandwidth, but this does not put any constraint onto the separation between frequencies associated to different entries of that vector, which may well be higher than the coherence bandwidth. Compared with the flat fading case, we now have the set of nuisance parameters $\left\{N_{k}\right\}_{k=1, \ldots, K}$ instead of only $N_{1}$, along with the set of unitary sub-band channel vectors $\left\{\mathbf{h}_{k}\right\}_{k=1, \ldots, K}$. While other models can in principle be assumed, the proposed piece-wise flat (within intervals $I_{k}$ indicated above) in the auxiliary frequency $v$ has the advantage of its mathematical simplicity and physical justification. In addition, it represents a natural extension of the full flat case (maximum signal covariance eigenvalue multiplicity) that we encounter for flat fading using Nyquist pulses. As will be

\footnotetext{
${ }^{13}$ Note that if the digital modulation were not a pulse-shaped digital modulations, the rank-1 preserving property would not hold.
}

clarified, this model provides as well a detector which is optimum at all SNR values, a property that we found for the flat case.

Using the above model, the SVD of the spectral coherence matrices within the range $v \in I_{k}$ can be expressed as

$$
\mathbf{S}_{\mathbf{y}}(v)=N_{0} \mathbf{I}+\gamma_{k} \mathbf{h}_{k} \mathbf{h}_{k}^{H}=\mathbf{U}_{\mathrm{k}} \boldsymbol{\Lambda}_{\mathrm{k}}(v) \mathbf{U}_{k}^{H}, \quad v \in I_{k},
$$

where

$$
\mathbf{U}_{k}=\left[\mathbf{h}_{k}, \mathbf{U}_{k}^{\perp}\right]
$$

and

$$
\boldsymbol{\Lambda}_{k}(v)=\left[\begin{array}{ccc}
N_{k} & \mathbf{0} & 0 \\
\mathbf{0} & N_{0} \mathbf{I}_{L-2} & \mathbf{0} \\
0 & \mathbf{0} & N_{0} \Upsilon(v)
\end{array}\right] .
$$

Note that $N_{k}=N_{0}+\gamma_{k}$ are free nuisance parameters at $\mathcal{H}_{1}$ while $N_{k}=N_{0}$ for all $k$ at $\mathcal{H}_{0}$. Splitting the integral of the log-likelihood into the intervals $I_{k}$, we can follow a similar development as in the flat fading case. By doing so (see appendix B for details), we obtain the following detector, which clearly constitutes a generalization of (37):

$$
\frac{1}{\left(\prod_{k=1}^{K} \lambda_{k}\right)^{1 / K}\left(1-\sum_{k=1}^{K} \lambda_{k}\right)^{\alpha}} \stackrel{\mathcal{H}_{1}}{\underset{\mathcal{H}_{0}}{\gtrless} \eta} \eta
$$

where

$$
\lambda_{k}=\lambda_{\max }\left\{\widetilde{\mathbf{B}}_{k}\right\}
$$

and

$$
\widetilde{\mathbf{B}}_{k}=\frac{\mathbf{B}_{k}}{\hat{P}_{T}} .
$$

Here, matrices $\mathbf{B}_{k}$ are short-band estimates of the spectral covariance matrix of the received signal, $\mathbf{S}_{y}(v)$, around frequencies $v_{k}$, given by

$$
\mathbf{B}_{k}=\int_{I_{k}} \mathbf{y}(v) \mathbf{y}^{H}(v) d v .
$$

The entries of these matrices can be seen as samples of the frequency-smoothed cyclic periodogram of the signal.

The interpretation of the obtained detector in (53) is as follows: while the first term of the denominator is a classical measure of inter-band sphericity, the second term of the denominator measures, contrarily, the intra-band sphericity, i.e., the lack of spectral correlation. The second term has no influence for $\alpha=0$, while its relative importance increases with the excess band. As a whole, the value of the detector increases when the measured spectrum is far from white and/or when high spectral correlation (i.e., high eigenvalue dispersion of sub-band matrices $\mathbf{B}_{k}$ ) is measured.

Remark 2. The problem of detecting low rank signals in the space domain using multiple antennas was developed by Ramirez et al. in [11], deriving a generalization of the sphericity ratio computed from the signal and noise eigenvalues of the covariance matrix. It can be easily seen that the generalized sphericity ratio test (see [11, Eq. (11)] reduces to a detector like that of (53) when the arithmetic mean of the noise eigenvalues is computed as a function of the total power and signal space power, and when the ratio between the signal rank and the total dimension (number of 
antennas) is specifically set to $1 /(1+\alpha)$. In the context of our problem, however, the advantage of the new sphericity test given in (53) is that, instead of requiring the SVD of a single large covariance matrix, it requires in contrast the simpler computation of maximum eigenvalues of several small-sized spectral covariance matrices. This is a consequence of both the asymptotic frequency-domain formulation and the sub-band rank-1 structure of cyclostationary processes. As a result the obtained detector looks only at the signal space, using the total power as a normalization factor.

Finally, it is worth mentioning that, although theoretical statistical analysis of detectors based on the estimation of a single maximum eigenvalue of a covariance matrix has been provided [13], the problem of theoretically characterizing the false and detection probabilities of the detector proposed in (53) is much more difficult because both arithmetic and geometric averages of eigenvectors are involved, and it is beyond the scope of this paper.

\section{A. Locally Most Powerful Invariant Test}

It has been recently proposed [37], [38] and proved [12] that for close hypothesis (i.e., low SNR), the LMPIT is given by the Frobenius norm of a normalized version of the autocorrelation matrix, which ignores the rank structure (i.e., the rank-1 structure in our case). This important result can be applied directly to yield the LMPIT for our problem, given as

$$
\sum_{k=1}^{K}\left\|\tilde{\mathbf{B}}_{k}\right\|_{F}^{2} \underset{\mathcal{H}_{0}}{\stackrel{\mathcal{H}_{1}}{\gtrless}} \eta,
$$

which can be rewritten simply as the aggregation of the modulus squared FS-CP, i.e., as

$$
\frac{\sum_{l=l_{0}}^{L-1} \sum_{n=-\left\lceil\frac{(L-l) K}{2}\right\rceil}^{\left\lceil\frac{(L-l) K}{2}\right\rceil}\left|\hat{S}_{x, M T, 1 /(K T)}^{l / T}\left(\frac{n}{K T}\right)\right|^{2}}{\hat{P}_{T}^{2}} \underset{\substack{\mathcal{H}_{0} \\ \underset{\mathcal{H}_{1}}{\gtrless} \eta,}}{\gtrless}
$$

where $l_{0}=0$. The numerator of the obtained detector coincides with the well-known multi-cycle spectral correlation magnitude detector (MCSCMD) proposed by Gardner [39, Eq. (34)] in the context of weak cyclostationary signals, where in general $l_{0}$ can take the values $l_{0}=0$ or $l_{0}=1$, depending on whether the stationary component is considered or not by the detector, respectively. The importance of this observation is twofold: on the one hand, it shows that the MCSCMD $\left(l_{0}=0\right)$ normalized by the squared total estimated power is in fact the LMPIT for the detection of a cyclostationary signal; on the other hand, this result is stated here because both the LMPIT and the MCSCMD $\left(l_{0}=1\right)$ will be considered for comparison purposes in the numerical results.

\section{Computational Complexity}

It is well known that cyclic spectral analysis is in general computationally expensive due to the potentially large number of spectral correlation computations, rather than the computation of the spectral components itself [40]. The proposed algorithms in this paper use a limited number of correlation computations, leading to improved complexity. To make this issue clear, this section evaluates the computational complexity as the number of complex multiplications involved in the proposed frequency-domain GLRT-cyc- $K$, as well as in the classical frequency-domain LMPIT- $K$ as a benchmark.

Given an observation sample size of $N_{s s} M$ samples, one needs $N_{s s} M \log _{2}\left(N_{s s} M\right)$ multiplications for the FFT, and $K L(L-1) / 2$ multiplications for the computation of the spectral matrices $\left\{\tilde{\mathbf{B}}_{k}\right\}$. While these computations are common in both algorithms, the improvement arises from the fact that the proposed GLRT-cyc- $K$ requires $K$ multiplications in the computation of maximum eigenvalues and their geometric mean, in front of the $K L(L-1) / 2$ multiplications in the computation of the Frobenious norms in LMPIT- $K$.

In conclusion, the complexity of the proposed GLRT-cyc- $K$ detector in terms of number of complex multiplications can be quantized as

$$
K L\left((Q-1) L^{2}+L+1\right) \approx Q K L^{2},
$$

which linearly increases with the number of iterations required by the Rayleigh quotient iteration (RQI) [41] method $(Q)$, linearly increases with the number of used sub-bands $(K)$, and quadratically increases with the dimension of the spectral covariance matrices $(L)$. Hence, it is worth emphasizing that $L$ solely depends on the excess band of the digital modulations, while it does not increase with the observation sample size.

Finally, it is further noted that the complexity of the GLRTcyc and the GLRT-cyc-ap detectors is nearly equal to that of the GLRT-cyc- $K$ by setting $K=1$, whereas the complexity of the MCSCMD- $K$ detector is nearly equal to that of the LMPIT- $K$ detector.

\section{NUMERICAL RESULTS}

We evaluate the performance of the proposed detectors by means of Monte Carlo simulations, using $10^{5}$ realizations per scenario. The transmission is modeled by $M$ QPSK symbols using RRC pulses. A tapped delay line channel model [7] with exponentially decaying power profile is considered with mean delay-spread $\tau$, such that it remains constant through a time of $M T$ seconds, and whose norm relative to the matched filter, i.e. $\int|H(f)|^{2}|P(f)|^{2} d f$, is kept constant in all realizations. For comparison, we consider the following six detectors.

1) $\operatorname{MCSCMD}-K$ with $l_{0}=1$, given by the numerator of (58).

2) GLRT-wss, given by the first term of (43), i.e,

$$
\frac{1}{\hat{P}_{T}} \operatorname{tr}(\mathbf{C}) \underset{\mathcal{H}_{0}}{\stackrel{\mathcal{H}_{1}}{\gtrless}} \eta .
$$

3) GLRT-cyc, given by (39).

4) GLRT-cyc-ap, given by (43).

5) LMPIT- $K$, given by (58).

6) The proposed GLRT-cyc- $K$, given by (53).

It is noted that the main purpose is to compare the performance of test 6) with other existing methods. Among them, test 5) is the main reference comparison, as it assumes identical prior knowledge and it is know to be the optimum low-SNR test. On the other hand, the comparison with the 


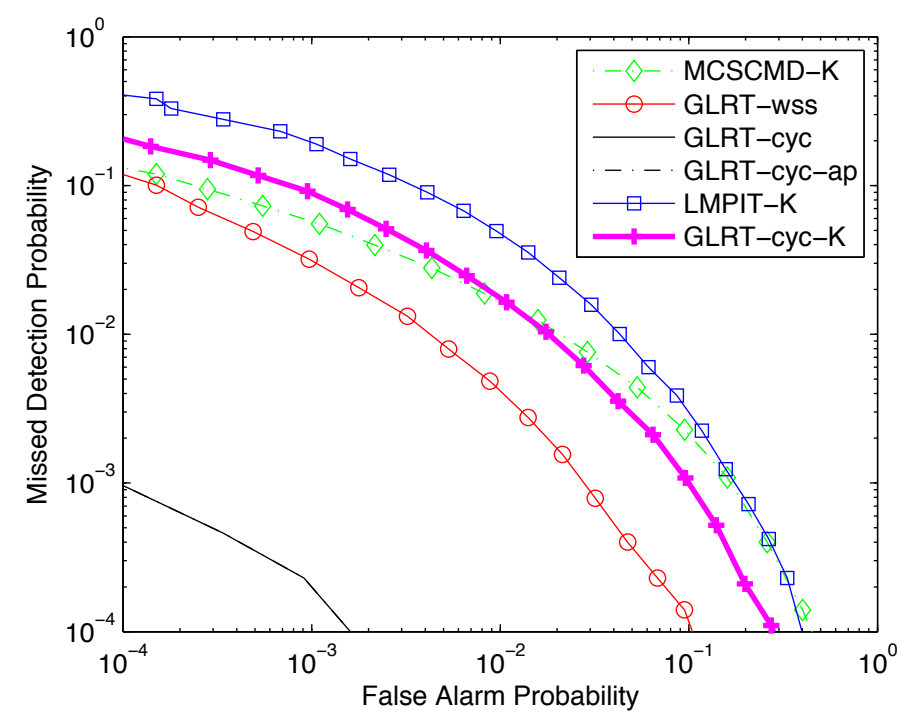

Fig. 1. ROC for the different detectors in the flat fading case $(\tau=0)$. $\alpha=0.8, S N R=-0.5 \mathrm{~dB}, M=256, K=8$.

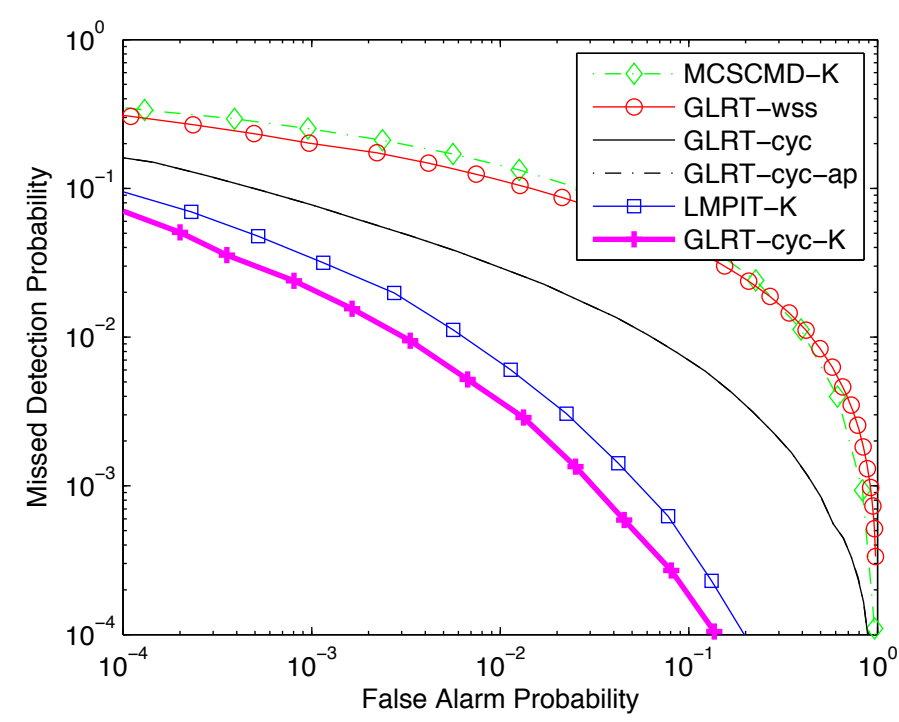

Fig. 2. ROC for the different detectors in the frequency-selective fading case $(\tau=1) . \alpha=0.8, S N R=-0.5 \mathrm{~dB}, M=256, K=8$.

exiting tests 4) and 3) is given to illustrate the lack of robustness in presence of a frequency-selective channel, and test 2) to illustrate the performance loss when cyclostationarity is not exploited. Finally, test 1) is the only one which is not invariant to scale (while all the remaining are), and its performance is included only as a reference and comparison of the proposed approach with a well-known method.

Fig. 1 shows the receiver operating characteristics (ROC), i.e., the missed detection probability versus the false alarm probability in the flat fading case $(\tau=0)$, with $\alpha=0.8$, $L=2$ and $\mathrm{SNR}=-0.5 \mathrm{~dB}$. It is straightforward to appreciate that the GLRT-cyc outperforms the other detectors, because it fully exploits the knowledge of the modulation structure of the PU.

Fig. 2 shows the ROC when the channel has a mean delayspread of $\tau=1$ symbol and illustrates the high sensitivity of

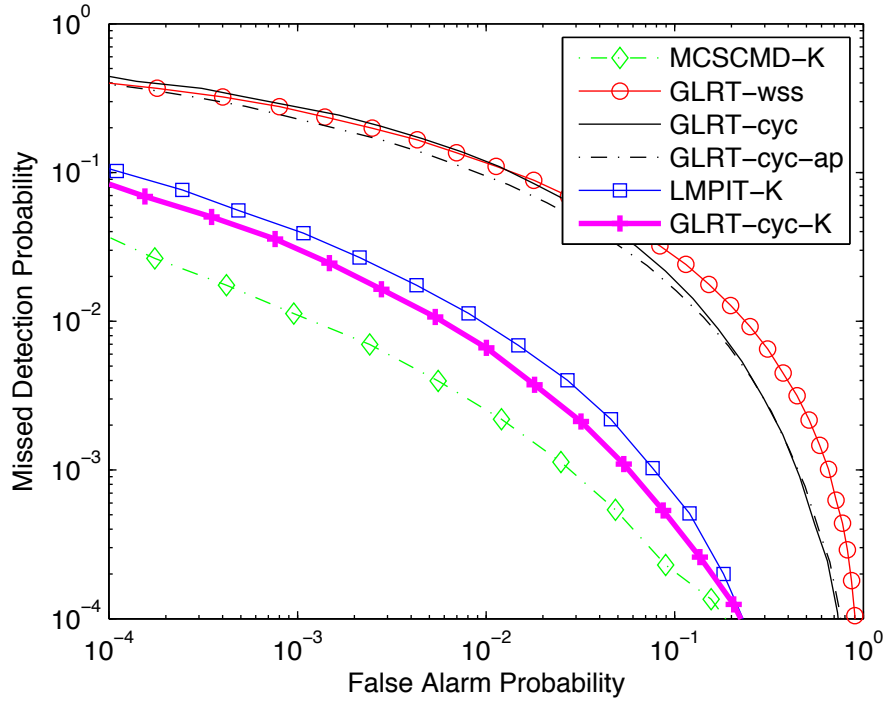

Fig. 3. ROC for the different detectors in the frequency-selective fading case $(\tau=2.5) . \alpha=3, S N R=-0.5 \mathrm{~dB}, M=256, K=8$.

GLRT-cyc in front of an unknown channel response. In contrast, the performance of the proposed GLRT-cyc- $K$ detector exhibits a robust behavior in detection performance in front of unknown frequency-selective channels.

Fig. 3 depicts the scenario simulated in Fig. 2 yet with a larger excess bandwidth. In particular, we set $\alpha=3$ by implementing the pulse shape

$$
p(t)=p_{0}(t) \frac{1+e^{j 2 \pi 2 t / T}}{\sqrt{2}},
$$

where $p_{0}(t)$ is a RRC pulse of unite roll-off. This situation requires the computation of spectral matrices of higher dimension $(L=4)$. As the excess bandwidth is larger, the GLRTcyc degradation is more relevant compared with that of Fig. 2. Even though, as the spectral correlation is richer (due to the increased excess bandwidth), the performance of the classical MCSCMD-K improves, and it even outperforms that of the LMPIT-K and GLRT-cyc-K. Thus, this scenario reflects the fact that the MCSCMD-K is making use of a known value of the noise power, while the remaining detectors are not, as they are invariant to scale. It is also seen that GLRT-cyc-ap has similar performance than that of the GLRT-cyc.

Fig. 4 shows the missed detection probability versus the SNR, where the thresholds have been set to satisfy a constant false alarm rate (CFAR) of 0.05 , and the remaining parameters are set as in Fig. 2. It is rapidly seen than the scaling of the detection performance with the SNR conditions, i.e., the error exponent, is significantly improved with the proposed GLRTcyc- $K$.

Fig. 5 summarizes the main advantage of the proposed GLRT-cyc- $K$ detector. It depicts the missed detection probability versus the mean delay spread of the channel, where the CFAR is set to 0.05 and the remaining parameters are set as in Fig. 2. It is appreciated that small values of delay spread cause a significant degradation in the detectors' performance. In contrast, the GLRT-cyc- $K$ tolerates higher delay spreads, showing a degradation only when the delay spread values 


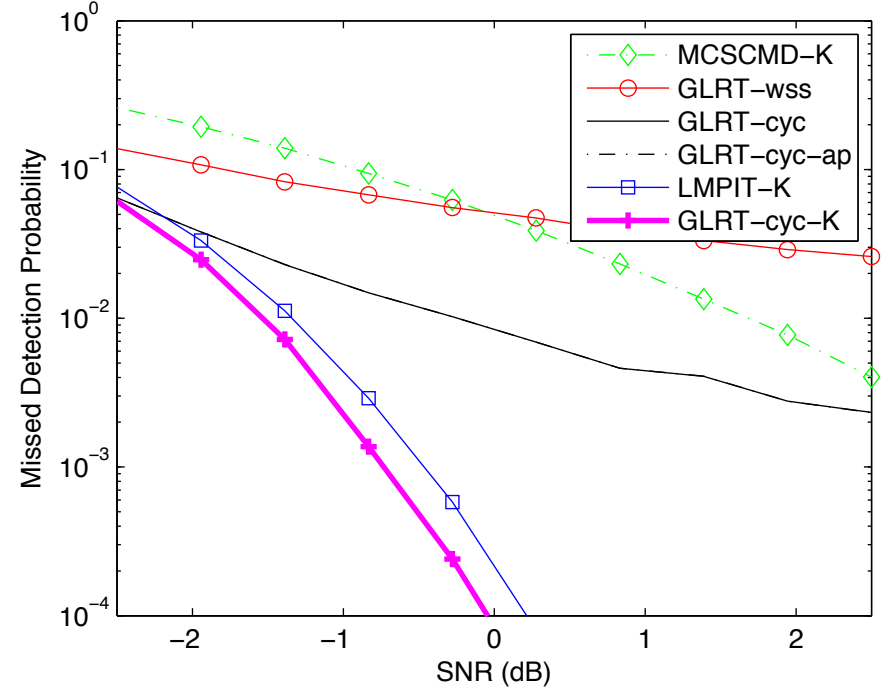

Fig. 4. Missed detection probability (for a false alarm probability of 0.05 ) versus SNR for different detectors. Same scenario as in Fig. 2.

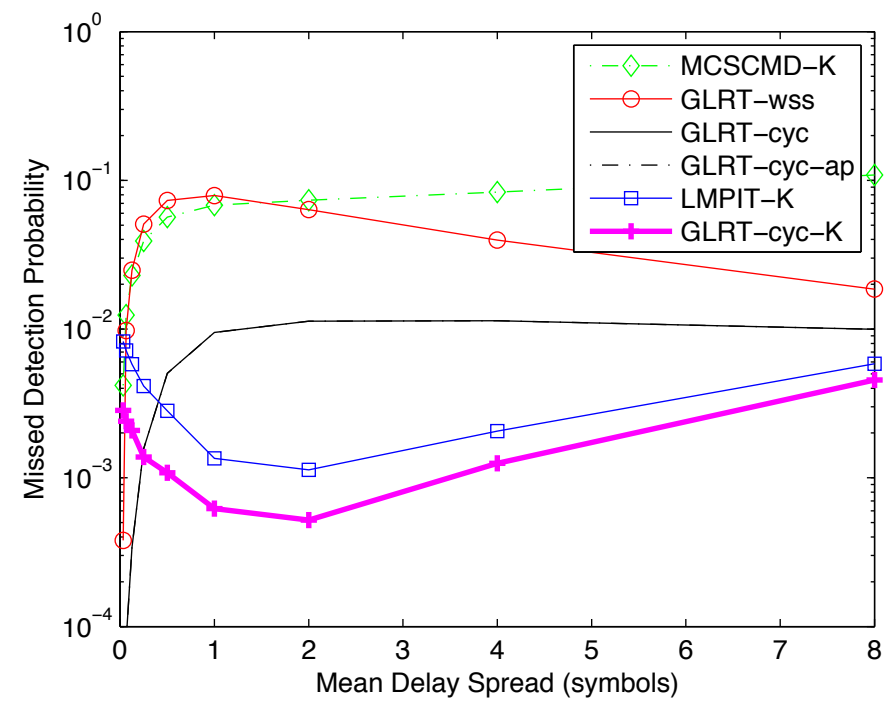

Fig. 5. Missed detection probability (for a false alarm probability of 0.05) versus mean delay spread $(\tau)$ for different detectors. Same scenario as in Fig. 2.

are of the same order as the number of sub-bands, i.e., $K$. Moreover, it is seen that the performance of the GLRT-cyc$K$ improves for small delay spread values. This is due to the fact that the GLRT-cyc- $K$ is partly sensitive to the lack of sphericity, which is measured by the geometrical mean term in the expression (53). In other words, in contrast to other cyclostationary based detectors, the proposed detector does not ignore the stationary spectral component of the signal, which is less flat in the presence of a frequency-selective channel.

Finally, Fig. 6 shows the effect of the block length $M$ on the missed detection probability for the GLRT-cyc- $K$ and LMPIT$K$ detectors at different values of SNR and a CFAR of 0.05 . From the illustrated curves, the SNR requirements in order to compensate small values of $M$ are appreciated.

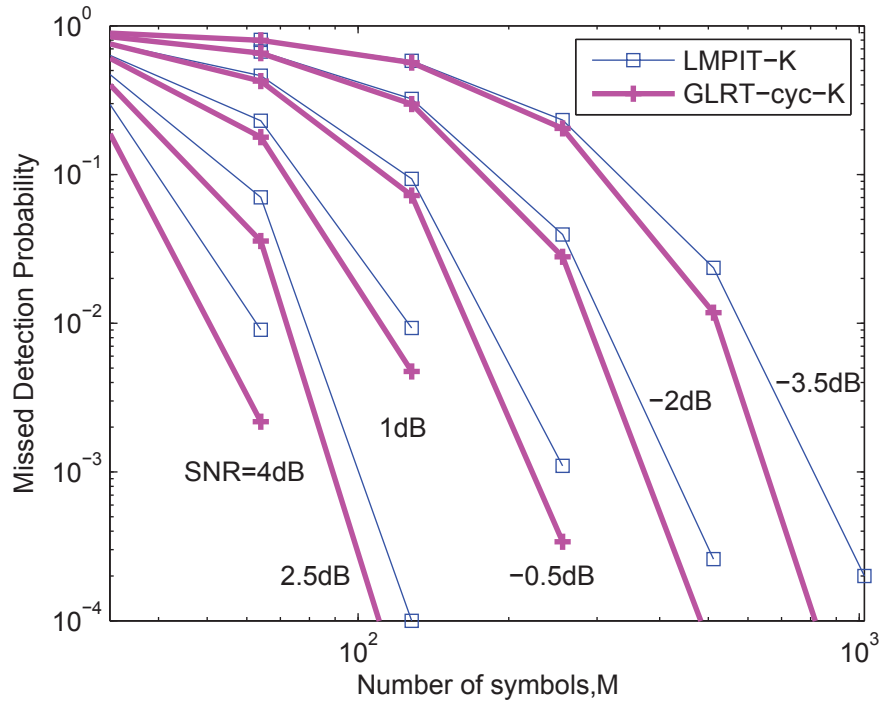

Fig. 6. Missed detection probability (for a false alarm probability of 0.05 ) number of symbols $(M)$ for the GRTL-cyc- $K$ and LMPIT- $K$ at different. Same scenario as in Fig. 2.

\section{CONCLUSIONS}

In this paper we have developed single-antenna, scaleinvariant detectors by using a new vectorial frequency-domain approach. The main advantage of this formulation is that it permits the use of mathematical tools typically developed in the array processing field, and it leads to new detection schemes with low computational complexity: they are based on estimating the cyclic spectral density (CSD) of the received signal (including the stationary component) and they require handling small-sized spectral covariance matrices. Moreover, only the computation of maximum eigenvalues of these matrices is required, instead of the singular value decomposition (SVD) of cumbersome covariance matrices required by other methods. The proposed detectors, which exploit both the stationary and cyclostationary component of the primary users' (PU) signal, are robust to the presence of an unknown frequency-selective channel, showing an improvement over other cyclostationary-based detection schemes. As the rank-1 structure of cyclostationary processes in the frequency-domain is exploited, the performance is still better than the classical (normalized) multi-cycle spectral correlation magnitude detector (MCSCMD) which ignores the rank-1 spectral correlation structure. Moreover, in our formulation, the MCSCMD detector (which is based on an aggregation of the modulus squared magnitude of the estimated CSD) is derived as the locally most powerful invariant test (LMPIT) of the problem. Using the proposed framework, future work will be focused to the development of detectors in the presence of unknown stationary interfering signals, as well as to the extension to the multiple antenna case, where the rank-1 structure in both space and frequency domains could be naturally exploited. Theoretical performance analysis of the proposed detectors in some specific scenarios will be also envisaged. 


\section{APPENDIX A}

DERIVATION OF (37)

Using the decomposition (34)-(36), we can write the loglikelihood function as

$$
\begin{aligned}
L(\mathbf{y})=- & \int_{I_{s}} \Lambda_{1}\left(v, N_{1}\right) d v \\
& \quad-\sum_{l=2}^{L-1} \int_{I_{s}} \Lambda_{l}\left(v, N_{0}\right) d v-\int_{I_{n}} \Lambda_{L}\left(v, N_{0}\right) d v
\end{aligned}
$$

where

$$
\Lambda_{l}(v, x)=\ln x+\frac{1}{x}\left|\mathbf{u}_{\varepsilon, l}^{H}(v) \mathbf{y}(v)\right|^{2},
$$

for $l=1, \ldots, L$, where $\mathbf{u}_{\varepsilon, l}(v)$ is the $l$-th column of matrix $\mathbf{U}_{\varepsilon}(v)$. Under $\mathcal{H}_{0}$, it is easily shown that the ML estimate of the noise floor simply becomes

$$
\begin{aligned}
\hat{N}_{0}^{0} & =\frac{T}{1+\alpha}\left(\hat{P}_{s, \varepsilon}+\hat{P}_{n}\right) \\
& =\frac{1}{B} \hat{P}_{T}
\end{aligned}
$$

where $\hat{P}_{T}$ is an estimate of the total received signal power given as

$$
\hat{P}_{T}=\int_{-B / 2}^{B / 2}|Y(f)|^{2} d f
$$

In (64), the estimates $\hat{P}_{s, \varepsilon}$ and $\hat{P}_{n}$ read

$$
\begin{aligned}
\hat{P}_{s, \varepsilon} & =\int_{I_{s}}\left|\mathbf{p}_{\varepsilon}^{H}(v) \mathbf{y}(v)\right|^{2} d v \\
& =\int_{I_{s}}\left|\mathbf{w}_{\varepsilon}^{H} \mathbf{z}(v)\right|^{2} d v,
\end{aligned}
$$

and

$$
\begin{aligned}
\hat{P}_{n} & =\sum_{l=2}^{L-1} \int_{I_{s}} \Lambda_{l}\left(v, N_{0}\right) d v+\int_{I_{n}} \Lambda_{L}\left(v, N_{0}\right) d v \\
& =\hat{P}_{T}-\hat{P}_{s, \varepsilon}
\end{aligned}
$$

respectively. Concerning $\mathcal{H}_{1}$, the ML estimates of $N_{1}$ and $N_{0}$ are given as [42]

$$
\hat{N}_{1, \varepsilon}^{1}=T \hat{P}_{s, \varepsilon}
$$

and

$$
\hat{N}_{0}^{1}=\frac{T}{\alpha} \hat{P}_{n}
$$

respectively. It is noted that the noise power estimation $\hat{P}_{n}$ based on integral in (69) for $L=2$ was proposed in [17] as an unbiased measure of the noise power in the presence of the signal of interest, with the goal of designing an SNR estimator based solely on second order statistics. After substitution in (62), maximizing with respect to the unknown $\varepsilon$ (as required by the GLRT approach, [15]) and removing additive constants, we obtain the detector

$$
\max _{\varepsilon}\left[\ln \frac{\hat{N}_{0}^{0}}{\hat{N}_{1, \varepsilon}^{1}}+\alpha \ln \frac{\hat{N_{0}^{0}}}{\hat{N_{0, \varepsilon}^{1}}}\right] \underset{\mathcal{H}_{0}}{\stackrel{\mathcal{H}_{1}}{\gtrless}} \eta,
$$

where $\eta$ is some threshold. The obtained expression of the GLRT can be further simplified as follows. We use (65)-(71) to write $\hat{N}_{0, \varepsilon}^{1}$ as a function of $\hat{N}_{0}^{0}$ and $\hat{N}_{1, \varepsilon}^{1}$ as

$$
\hat{N}_{0, \varepsilon}^{1}=\frac{(1+\alpha) \hat{N}_{0}^{0}-\hat{N}_{1, \varepsilon} .}{\alpha} .
$$

The above substitution is important because it implies that estimation of the power at the noise subspace will never be required, being the total and the signal subspace powers the sufficient statistics for the detector. After some trivial manipulation, we obtain (37).

\section{APPENDIX B}

\section{DERIVATION OF (53)}

Splitting the integral of the likelihood function in subintervals

$$
\begin{aligned}
& L(\mathbf{y})=-\frac{\left|I_{s}\right|}{K} \sum_{k} \ln N_{k}- \\
& \sum_{k} \frac{1}{N_{k}} \hat{P}_{s, k}-I_{n} \ln N_{0}-\frac{1}{N_{0}} \sum_{k} \hat{P}_{n, k},
\end{aligned}
$$

where

$$
\begin{aligned}
\hat{P}_{s, k} & =\int_{I_{k}}\left|\mathbf{h}_{k}^{H}(v) \mathbf{y}(v)\right|^{2} d \nu=\mathbf{h}_{k}^{H} \hat{\mathbf{B}}_{k} \mathbf{h}_{k} \\
\hat{P}_{n, k} & =\int_{I_{k}}\left|\mathbf{u}_{k}^{\perp H}(v) \mathbf{y}(v)\right|^{2} d \nu .
\end{aligned}
$$

Under $\mathcal{H}_{0}$, the ML estimate of the noise floor is given by

$$
\begin{aligned}
\hat{N}_{0}^{0} & =\frac{1}{I_{s}+I_{n}}\left(\hat{P}_{s}+\hat{P}_{n}\right) \\
& =\frac{1}{B} \int_{-B / 2}^{B / 2}|Y(f)|^{2} d f \\
& =\frac{1}{B} \hat{P}_{T}
\end{aligned}
$$

where

$$
\hat{P}_{s}=\sum_{k} \hat{P}_{s, k}
$$

and

$$
\hat{P}_{n}=\sum_{k} \hat{P}_{n, k}
$$

Concerning $\mathcal{H}_{1}$, the ML estimates of $\left\{N_{k}\right\}$ and $N_{0}$ are given as

$$
\hat{N}_{k}=\frac{1}{I_{s} / K} \hat{P}_{s, k}
$$

and

$$
\hat{N}_{0}^{1}=\frac{1}{I_{n}} \hat{P}_{n}=\frac{(1+\alpha) \hat{N}_{0}^{0}-\frac{1}{K} \sum_{k} \hat{N}_{k}}{\alpha}
$$

Substituting these estimates to the likelihood function and forming the GLRT yields

$$
\max _{\left\{\mathbf{h}_{k}\right\}} \frac{1}{\left(\prod_{k=1}^{K} \lambda_{k}\right)^{1 / K}\left(1-\sum_{k=1}^{K} \lambda_{k}\right)^{\alpha}} \stackrel{\mathcal{H}_{1}}{\underset{\mathcal{H}_{0}}{\gtrless}} \eta,
$$

with

$$
\lambda_{k}=\frac{\mathbf{h}_{k}^{H} \mathbf{B}_{k} \mathbf{h}_{k}}{\hat{P}_{T}}
$$


Maximizing with respect to the $\left\{\mathbf{h}_{k}\right\}_{k=1, \ldots, K}$ under the constraint $\left\{\left\|\mathbf{h}_{k}\right\|^{2}=1\right\}_{\}_{k=1, \ldots, K}}$ yields (53).

\section{REFERENCES}

[1] E. Axell, G. Leus, E. G. Larsson, and H. V. Poor, "Spectrum sensing for cognitive radio: State-of-the-art and recent advances," IEEE Signal Process. Magazine, vol. 29, no. 3, pp. 101-116, Mar. 2012.

[2] R. Tandra and A. Sahai, "SNR walls for signal detection," IEEE J. Sel. Topics Signal Process., vol. 2, no. 1, pp. 4-17, Feb. 2008.

[3] G. Huang and J. K. Tugnait, "On cyclostationarity based spectrum sensing under uncertain Gaussian noise," IEEE Trans. Signal Process., vol. 61, no. 8, pp. 2042-2054, Apr. 2013.

[4] W. A. Gardner, "Signal interception: A unifying theoretical framework for feature detection," IEEE Trans. Commun., vol. 36, no. 8, pp. 897906, Aug. 1986.

[5] D. Cabric, "Addressing the feasibility of cognitive radios," IEEE Signal Process. Magazine, vol. 25, no. 6, pp. 85-93, Nov. 2008.

[6] Z. Quan, W. Zhang, S. J. Shellhammer, and A. H. Sayed, "Optimal spectral feature detection for spectrum sensing at very low SNR," IEEE Trans. Commun., vol. 59, no. 1, pp. 201-212, Jan. 2011.

[7] E. Biglieri, J. Proakis, and S. Shamai, "Fading channels: informationtheoretic and communications aspects," IEEE Trans. Inf. Theory, vol. 44, no. 6, pp. 2619-2692, Oct. 1998.

[8] R. Tandra and A. Sahai, "SNR walls for feature detectors," in IEEE Int. Symp. on New Front. in Dyn. Spec. Access, Apr. 2007.

[9] E. Axell and E. G. Larsson, "Multiantenna spectrum sensing of a secondorder cyclostationary signal," in IEEE Int. Workshop Computat. Adv. Multi-Sens. Adapt. Process. (CAMSAP), Dec. 2011.

[10] R. Zhang, T. J. Lim, Y.-C. Liang, and Y. Zeng, "Multi-antenna based spectrum sensing for cognitive radios: A GLRT approach," IEEE Trans. Commun., vol. 58, no. 1, pp. 84-88, Jan. 2010.

[11] D. Ramirez, G. Vazquez-Vilar, R. López-Valcarce, J. Vía, and I. Santamaría, "Detection of rank- $P$ signals in cognitive radio networks with uncalibrated multiple antennas," IEEE Trans. Signal Process., vol. 59, no. 8, pp. 3764-3774, Aug. 2011.

[12] D. Ramirez, J. Via, I. Santamaria, and L. L. Scharf, "Locally most powerful invariant tests for correlation and sphericity of Gaussian vectors," IEEE Trans. Inf. Theory, vol. 59, no. 4, pp. 2128-2141, Apr. 2013.

[13] O. Besson, S. Kraut, and L. L. Scharf, "Detection of an unknown rankone component in white noise," IEEE Trans. Signal Process., vol. 54 no. 7, pp. 2835-2839, Jul. 2006.

[14] A. I. X. Gong and G. Ascheid, "GLRT-based cooperative spectrum sensing for cognitive radio with rank information," in IEEE Stat. Signal Process. Workshop (SSP), Jun. 2011.

[15] S. M. Kay, Fundamentals of Statistical Signal Processing: Detection Theory. New York: Prentice-Hall, 1998, vol. 2.

[16] W. Zhang, H. V. Poor, and Z. Quan, "Frequency-domain correlation: An asymptotically optimum approximation of quadratic likelihood ratio detectors," IEEE Trans. Signal Process, vol. 58, no. 3, pp. 969-979, Mar. 2010

[17] J. Riba, J. Villares, and G. Vazquez, "A nondata-aided SNR estimation technique for multilevel modulations exploiting signal cyclostationarity," IEEE Trans. Signal Process., vol. 58, no. 11, pp. 5767-5778, Nov. 2011

[18] "Digital Video Broadcasting (DVB); Frame structure, channel coding and modulation for a Second Generation Digital Terrestrial Television Broadcasting System (DVB-T2), EN 302755 V.1.1.1," Sept. 2009.

[19] "Digital Video Broadcasting (DVB); Second Generation Framing Structure, Channel Coding and Modulation Systems for Broadcasting, Interactive Services, News Gathering and Other Broadband Satellite Applications, EN 300307 V1.1.2," Jun. 2006.

[20] M. Sadler and A. V. Dandawate, "Nonparametric estimation of the cyclic cross spectrum," IEEE Trans. Inf. Theory, vol. 44, no. 1, pp. 351-358, Jan. 1998.

[21] P. J. Schreier and L. L. Scharf, Statistical Signal Processing of ComplexValued Data. The Theory of Improper and Noncircular Signals. Cambridge University Press, 2010.

[22] W. A. Gardner, Statistical Spectral Analysis: A Nonprobabilistic Theory. Prentice-Hall, 1986.

[23] E. Serpedin, F. Panduru, I. Sari, and G. B. Giannakis, "Bibliography on cyclostationarity," Signal Process., vol. 85, no. 12, pp. 2233-2303, 2005.

[24] W. A. Gardner, A. Napolitano, and L. Paura, "Cyclostationarity: Half a century of research,” Signal Process., vol. 86, no. 4, pp. 639-697, Apr 2006.
[25] D. R. Brillinger, Time Series: Data Analysis and Theory. San Francisco: Holden-Day, 1981, (Section 4.4: asymptotic distribution of the finite Fourier transform).

[26] J. Villares and G. Vazquez, "The Gaussian assumption in second-order estimation problems in digital communications," IEEE Trans. Signal Process., vol. 55, no. 10, pp. 4994-5002, Oct. 2007.

[27] A. V. Dandawate and G. B. Giannakis, "Statistical tests for presence of cyclostationarity," IEEE Trans. Signal Process., vol. 42, no. 9, pp. 2355-2369, Sep. 1994.

[28] J. Lunden, V. Koivunen, A. Huttunen, and H. V. Poor, "Collaborative cyclostationary spectrum sensing for cognitive radio systems," IEEE Trans. Signal Process., vol. 57, no. 11, pp. 4182-4195, Nov. 2009.

[29] H. V. Poor, An Introduction to Signal Detection and Estimation, 2nd ed. New York: Springer-Verlag, 1994.

[30] E. Axell and E. G. Larsson, "A unified framework for GLRT-based spectrum sensing of signals with covariance matrices with known eigenvalue multiplicities," in Proc. IEEE Int. Conf. on Acoust., Speech and Signal Process. (ICASSP), May 2011.

[31] _ , "Eigenvalue-based spectrum sensing of orthogonal space-time block coded signals," IEEE Trans. Signal Process., vol. 60, no. 12, pp. 6724-6728, Dec. 2012.

[32] A. Taherpour, M. Nasiri-Kenari, and S. Gazor, "Multiple antenna spectrum sensing in cognitive radios," IEEE Trans. Wireless Comm., vol. 9, no. 2, pp. 814-823, Feb. 2010.

[33] S. Haykin, D. J. Thomson, and J. H. Reed, "Spectrum sensing for cognitive radio," Proc. IEEE, vol. 97, no. 5, pp. 849-877, May 2009.

[34] M. Oerder and H. Meyr, "Digital filter and square timing recovery," IEEE Trans. Commun., vol. 36, no. 5, pp. 605-612, May. 1988.

[35] U. Mengali and A. N. D'Andrea, Synchronization Techniques for Digital Receivers. Plenum Press, 1997.

[36] J. A. Lopez-Salcedo and G. Vazquez, "Asymptotic equivalence between the unconditional maximum likelihood and the square-law nonlinearity symbol timing estimation," IEEE Trans. Signal Process., vol. 54, no. 1, pp. 244-257, Jan. 2006.

[37] A. Leshem and A.-J. V. der Veen, "Multichannel detection of Gaussian signals with uncalibrated receivers," IEEE Signal Process. Lett., vol. 8, no. 4, pp. 120-122, Apr. 2001

[38] J. Font-Segura, J. Riba, J. Villares, and G. Vazquez, "Quadratic sphericity test for blind detection over time-varying frequency-selective channels," in IEEE Int. Conf. on Acoust., Speech and Signal Process. (ICASSP), May 2013

[39] W. A. Gardner and C. M. Spooner, "Detection and source location of weak cyclostationary signals: simplifications of the maximum-likelihood receiver," IEEE Trans. Commun., vol. 41, no. 6, pp. 905-916, Jun. 1993.

[40] R. S. Roberts, W. A. Brown, and H. H. Loomis, "Computationally efficient algorithms for cyclic spectral analysis," IEEE Signal Process. Magazine, vol. 8, no. 2, pp. 38-49, Apr. 1991.

[41] G. H. Golub and C. F. V. Loan, Matrix computations. Johns Hopkins University Press, 1996

[42] T. W. Anderson, "asymptotic theory for principal component analysis," The Annals of Math. Stat., vol. 34, no. 1, pp. 122-148, Mar. 1963. 